Check for updates

Cite this: RSC Adv., 2018, 8, 27246

Received 20th March 2018

Accepted 3rd July 2018

DOI: $10.1039 / \mathrm{c} 8 \mathrm{ra02448 \textrm {k }}$

rsc.li/rsc-advances

\title{
A facile and general approach for the direct fabrication of N-rGO-metal(metal oxides)-Pt composites as electrocatalyst for oxygen reduction reactions $\dagger$
}

\begin{abstract}
Xiaohong Chen, (D) a Zhaoyang Ning, ${ }^{\mathrm{C}}$ Zongshang Zhou, ${ }^{\mathrm{b}}$ Xundao Liu, ${ }^{\text {a }}$ Jianlong Lei, ${ }^{\mathrm{a}}$ Supeng Pei ${ }^{\star a b}$ and Yongming Zhang ${ }^{\star a}$

Nitrogen-doped reduced graphene oxide-metal(metal oxides) nanoparticle (N-rGO-M(MO) NPs, $M=F e$, $\mathrm{MO}: \mathrm{M}=\mathrm{Co}, \mathrm{Mn})$ composites were prepared through a facile and general method at high temperature $(800$ $\left.{ }^{\circ} \mathrm{C}\right) . \mathrm{M}(\mathrm{MO})$ were well-dispersed and tightly anchored on graphene sheets, which were doped with nitrogen simultaneously and further loaded with Pt nanoparticles. Those results showed a more positive onset potential, higher cathodic density, and higher electron transfer number for the ORR in alkaline media. Furthermore, N-rGO-metal(metal oxides)-Pt (N-rGO-M(MO)-Pt) nanoparticles show better durability than the commercial $\mathrm{Pt} / \mathrm{C}$ catalyst, and can be used as promising potential materials in practical applications.
\end{abstract}

\section{Introduction}

The electrocatalyst for the oxygen reduction reaction (ORR) has recently attracted a tremendous amount of attention, due to the significance in various renewable energy applications, such as fuel cells and metal-air batteries. ${ }^{1-8}$ Recently, ORR catalyzed by Pt-based catalysts has demonstrated a high onset potential and fast kinetics and a four-electron transfer process. ${ }^{9-14}$ Meanwhile, heterogeneous atom doping could also potentially produce localized highly active regions of graphene, while the electric conductivity of the functionalized graphene is improved. ${ }^{15-23}$

According to the previous reports, ${ }^{24-27}$ N-rGO-M(MO) composites were synthesized by different methods, such as precursor pyrolysis, the hydrothermal method, the template method and others. Shihe Yang reported cobalt-embedded nitrogen doped carbon nanotubes for OER/ORR bifunctional catalysts, designed and prepared using CoPt as the Co and nitrogen sources, which is particularly suitable for synthesizing

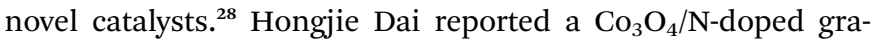
phene composite by a two-step method as one of the rare and

${ }^{a}$ School of Chemistry and Chemical Engineering, Shanghai Key Lab of Electrical Insulation and Thermal Aging, Shanghai Electrochemical Energy Devices Research Center, Shanghai Jiao Tong University, No. 800 Dongchuan Rd., Minhang District, Shanghai 200240, China. E-mail: peisupeng@126.com; ymzsjtu@gmail.com

${ }^{b}$ School of Chemical and Environmental Engineering, Shanghai Institute of Technology, Shanghai 201418, China

${ }^{c}$ Shandong Fuyuan Energy Conservation and Environmental Protection Engineering Co., Ltd., Jinan, 250000, China

$\dagger$ Electronic supplementary information (ESI) available. See DOI: $10.1039 / \mathrm{c} 8 \mathrm{ra} 02448 \mathrm{k}$ highest performance bi-functional catalysts for ORR and water oxidation/OER ${ }^{29} \mathrm{Fu}$ et al. established a soft template F-127 and hard template tellurium nanowires to produce a onedimensional hierarchical porous $\mathrm{PtCu}$ alloy nanostructure with large mesopores, which exhibits enhanced electrocatalytic performance for the ORR in acid media. ${ }^{30}$ Therefore, a more efficient and convenient procedure would be desirable for improving efficiency. It was believed that combining platinum, transition metal oxides and conductive nanocarbon materials such as graphene and carbon nanotubes is an effective approach for enhancing the catalytic activity of oxides. This is attributed to an increased number of available active sites and providing efficient charge transport channels.

Herein, we reported a facile, yet general approach to prepare $\mathrm{N}-\mathrm{rGO}-\mathrm{M}(\mathrm{MO})$ nanoparticles, which was coreduced from metal salt and melamine at a high temperature $\left(800^{\circ} \mathrm{C}\right)$. Those results indicated that the $\mathrm{M}(\mathrm{MO})$ NPs were strongly bound to uniformly distributed on the N-rGO, while Pt nanoparticles could be easily

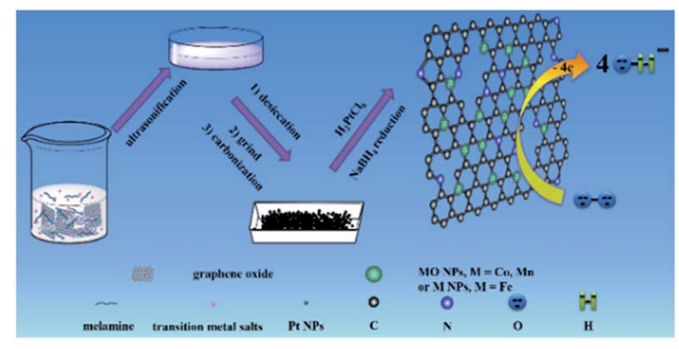

Scheme 1 Fabrication procedure for N-rGO-Co/CoO-Pt, N-rGO$\mathrm{Fe}-\mathrm{Pt}$ and $\mathrm{N}-\mathrm{rGO}-\mathrm{Mn}$ oxides-Pt composites. 
loaded on the N-rGO-M(MO) materials (Scheme 1). Remarkably, the N-rGO-M(MO)-Pt composites showed a much more positive ORR onset potential $(66,140$ and $253 \mathrm{mV}$ ) than that of $20 \% \mathrm{Pt} / \mathrm{C}$, suggesting synergistic ORR catalytic activity of Pt, M(MO) and N-rGO in the composites. Therefore, the half-wave potential, $J_{\mathrm{k}}$ and durability of N-rGO-M(MO)-Pt composites are demonstrated.

\section{Experimental}

\subsection{Synthesis of nitrogen-doped reduced graphene (N-rGO),} N-rGO-Co/CoO, N-rGO-Fe and N-rGO-Mn oxides

The main text of the article should appear here with headings as appropriate. We used high-purity argon as protective ambient to anneal a mixture of GO, melamine and $\mathrm{Co}(\mathrm{Ac})_{2}$. Detailed procedure is as follows: GO powder, melamine and $\mathrm{Co}(\mathrm{Ac})_{2}$ were ground together in an agate mortar for about $5 \mathrm{~min}$, and the mixture was then placed in the center of a quartz tube with a flow of argon. Before the furnace was heated to $800{ }^{\circ} \mathrm{C}$, Ar gas was flowed for about $20 \mathrm{~min}$. When the center of the furnace reached the designed reaction temperature $\left(\right.$ e.g., $\left.800{ }^{\circ} \mathrm{C}\right)$, the mixture was annealed for $1 \mathrm{~h}$ at this temperature. After that, the sample was cooled to room temperature under $\mathrm{Ar}$ ambient. Finally, the products were taken out of the quartz tube. N-rGO, N-rGO-Co/CoO, N-rGO-Fe and N-rGO-Mn oxides was synthesized from GO using a similar procedure.

\subsection{Synthesis of N-rGO-Co/CoO-Pt, N-rGO-Fe-Pt and N- rGO-Mn oxides-Pt composites}

$20 \mathrm{~mL}$ of $\mathrm{N}$-rGO-Co/CoO $\left(1 \mathrm{mg} \mathrm{mL}^{-1}\right)$ aqueous dispersion was prepared by ultrasonification, into which $\mathrm{H}_{2} \mathrm{PtCl}_{6}$ aqueous solution $\left(9.2 \mathrm{~mL}, 4 \mathrm{mg} \mathrm{mL}{ }^{-1}\right)$ was dropped under nitrogen. Then $100 \mathrm{~mL}$ of $\mathrm{NaBH}_{4}\left(2 \mathrm{mg} \mathrm{mL}{ }^{-1}\right)$ was added dropwise. After stirring for $2 \mathrm{~h}$ and standing overnight, the resulting $\mathrm{N}-\mathrm{rGO}-\mathrm{Co} /$ CoO-Pt catalyst was filtrated and washed with DIW several times. The resulting product was dried in vacuum at $40{ }^{\circ} \mathrm{C}$ for 24 h. For comparison, N-rGO-Fe-Pt and N-rGO-Mn oxides-Pt was prepared according to the similar procedures. Note: the $\mathrm{Pt}$ loading of the catalysts is $10 \mathrm{wt} \%$ unless specified.

\subsection{Preparation of Pt/C, N-rGO-Co/CoO, N-rGO-Co/CoO-Pt, $\mathrm{N}$-rGO-Fe-Pt and N-rGO-Mn oxides-Pt modified electrode}

Prior to use, glassy carbon electrode (GCE, $\varphi=3 \mathrm{~mm}$ ) was polished with $0.05 \mu \mathrm{m}$ gamma alumina powders, then rinsed thoroughly with ethanol and water in an ultrasonic bath to remove any alumina residues, and finally dried at room temperature. $4 \mu \mathrm{L}$ of $1 \mathrm{mg} \mathrm{mL}{ }^{-1}$ sonicated $20 \%$ commercial Pt$\mathrm{C}, \mathrm{N}-\mathrm{rGO}-\mathrm{Co} / \mathrm{CoO}, \mathrm{N}-\mathrm{rGO}-\mathrm{Co} / \mathrm{CoO}-\mathrm{Pt}, \mathrm{N}-\mathrm{rGO}-\mathrm{Fe}-\mathrm{Pt}$ and $\mathrm{N}$ rGO-Mn oxides-Pt with ethanol and water suspension was dropped on the pretreated bare GCE or GC disk using a micropipet tip and dried in air.

\subsection{Cyclic voltammograms (CV) measurement}

A conventional cell with a three-electrode configuration was served throughout this work. CV measurement was conducted at $25{ }^{\circ} \mathrm{C}$ using an Autolab PGSTAT302 (Metrohm) electrochemical test system using saturated $\mathrm{Ag} / \mathrm{AgCl}$ as the reference electrode, a Pt wire as the counter electrode and the sample modified glassy carbon electrode as the working electrode. 0.1 $\mathrm{M} \mathrm{KOH}$ aqueous solution was used as the electrolyte, which was saturated with $\mathrm{O}_{2}$ by bubbling it prior to the start of each experiment. A flow of $\mathrm{O}_{2}$ was maintained over the electrolyte during the recording of CVs in order to ensure continuous $\mathrm{O}_{2}$ saturation. The working electrode was cycled at least 5 times before data recorded at a scan rate of $10 \mathrm{mV} \mathrm{s}^{-1}$. In control experiments, CV measurements were also performed under $\mathrm{N}_{2}$ atmosphere. In all electrochemical measurements, $0.1 \mathrm{M} \mathrm{KOH}$ aqueous solution saturated with nitrogen or oxygen was used as the electrolyte.

\subsection{Rotating disk electrode (RDE) measurement}

For the RDE measurement, catalyst inks were prepared by the same method as that of $\mathrm{CV}$ analysis described above. $4 \mu \mathrm{L}$ of $5 \mathrm{mg} \mathrm{mL}{ }^{-1}$ ink was loaded on a glassy carbon $\operatorname{RDE}(\varphi=3 \mathrm{~mm})$. The working electrode was cathodically scanned at a rate of $10 \mathrm{mV} \mathrm{s}^{-1}$ with varying rotating speed from 400 to $2000 \mathrm{rpm}$. The ORR current was determined by subtracting the $\mathrm{N}_{2}$ current from the $\mathrm{O}_{2}$ current. Koutecky-Levich plots $\left(J^{-1}\right.$ vs. $\left.\omega^{-1 / 2}\right)$ were analyzed at various electrode potentials. The slopes of their best linear fit lines were used to calculate the number of electrons transferred $(n)$ on the basis of the Koutecky-Levich equation:

$$
\frac{1}{J}=\frac{1}{J_{\mathrm{L}}}+\frac{1}{J_{\mathrm{K}}}=\frac{1}{B \omega^{1 / 2}}+\frac{1}{J_{\mathrm{K}}}
$$

$$
B=0.62 n F C_{0}\left(D_{0}\right)^{2 / 3} v^{-1 / 6} J_{\mathrm{k}}=n F k C_{0}
$$

where $J$ is the measured current density, $J_{\mathrm{K}}$ and $J_{\mathrm{L}}$ are the kinetic and diffusion limiting current densities, $\omega$ is the angular velocity, $n$ is transferred electron number, $F$ is the Faraday constant (96485 $\mathrm{C} \mathrm{mol}^{-1}$ ), $C_{0}$ is the bulk concentration of $\mathrm{O}_{2}$ $\left(1.2 \times 10^{-3} \mathrm{~mol} \mathrm{~cm}^{-3}\right), D_{0}$ is the diffusion coefficient of $\mathrm{O}_{2}(1.9$ $\left.\times 10^{-5}\right), v$ is the kinematic viscosity of the electrolyte $\left(0.01 \mathrm{~m}^{2}\right.$ $\mathrm{s}^{-1}$ ), and $k$ is the electron-transfer rate constant.

\subsection{Materials and methods}

The main text of the article should appear here with headings as appropriate. Melamine, $\mathrm{Co}(\mathrm{Ac})_{2}, \mathrm{FeCl}_{3}$ and $\mathrm{MnCl}_{2}$ were bought from Sinopharm Chemical Reagent CO. Ltd. (China). Other chemicals such as ethanol, $\mathrm{KOH}$ and $N, N$-dimethylformamide (DMF) bought from Shanghai Lingfeng Chemical Reagent CO. Ltd., were all of analytical grade and used as received. All solutions used in the electrochemical experiments were freshly prepared with Millipore water (resistivity $\sim 18.2 \mathrm{M} \Omega$ ). The morphology of N-rGO-Co/CoO-Pt, N-rGO-Fe-Pt and N-rGO-Mn oxides-Pt was characterized by transmission electron microscopy (TEM) and scanning electron microscopy (SEM) using JEM2010HT and JEOL2100F (both from Electron Optics Laboratory Co., Ltd., Japan), respectively. Powder X-ray diffraction (XRD) analyses were performed on a diffractometer (Bruker, German, APLX-DUO) with $\mathrm{Cu} \mathrm{K} \alpha$ radiation. Raman spectra were recorded 
using a Thermo Fisher H31XYZE-US with an excitation wavelength of $532 \mathrm{~nm}$. X-ray photoelectron spectroscopy (XPS, $\mathrm{K}_{\alpha}$ ) analyses were carried out on an AXIS UltraDLD X-ray photoelectron spectrometer system equipped with $\mathrm{Al}$ radiation as a probe, and the analysis spot size was $400 \mu \mathrm{m}$ in diameter.

All electrochemical measurements were performed on an Autolab PGSTAT302N (Metrohm, Switzerland) electrochemical test system. Cyclic voltammograms were collected in a threeelectrode system (GCE or modified GCE as the working electrode, a Pt wire as counter electrode, and an $\mathrm{Ag} / \mathrm{AgCl}$ as the reference) at room temperature. For RDE measurements, a glassy carbon rotating disk electrode was used as the working electrode. Linear sweep voltammetry was performed at the GC disk electrode. In all of the electrochemical measurements, $0.1 \mathrm{M} \mathrm{KOH}$ aqueous solution saturated with nitrogen or oxygen was used as the electrolyte.

\section{Results and discussion}

The composition and crystal structure of $\mathrm{N}-\mathrm{rGO}-\mathrm{Co} / \mathrm{CoO}, \mathrm{N}-$ rGO-Co/CoO-Pt, N-rGO-Fe, N-rGO-Fe-Pt, N-rGO-Mn oxides and N-rGO-Mn oxides-Pt composite nanofibers were firstly characterized by XRD and Raman (Fig. 1 and 2). The XRD pattern confirmed the formation of Co (JCPDS no. 15-0806) and CoO (JCPDS no. 65-5474) and N-rGO in the composites (Fig. 1A). The $\mathrm{C}$ (002) diffraction peak in $\mathrm{N}-\mathrm{rGO}-\mathrm{Co} / \mathrm{CoO}$ seems to be broader and faint, indicating the relatively less dense packing of graphene sheets due to $\mathrm{N}$-induced structural defects. Meanwhile, the XRD pattern confirmed the formation of $\mathrm{Fe}_{5} \mathrm{C}_{2}$ (JCPDS no. 35-0772), $\mathrm{MnOOH}$ (JPCDS no. 41-1379) and $\mathrm{Mn}_{2} \mathrm{O}_{3}$ (JPCDS no. 24-0508) in the composites (Fig. 1C and E). The characteristic diffraction peaks indicated that Fe nanoparticles

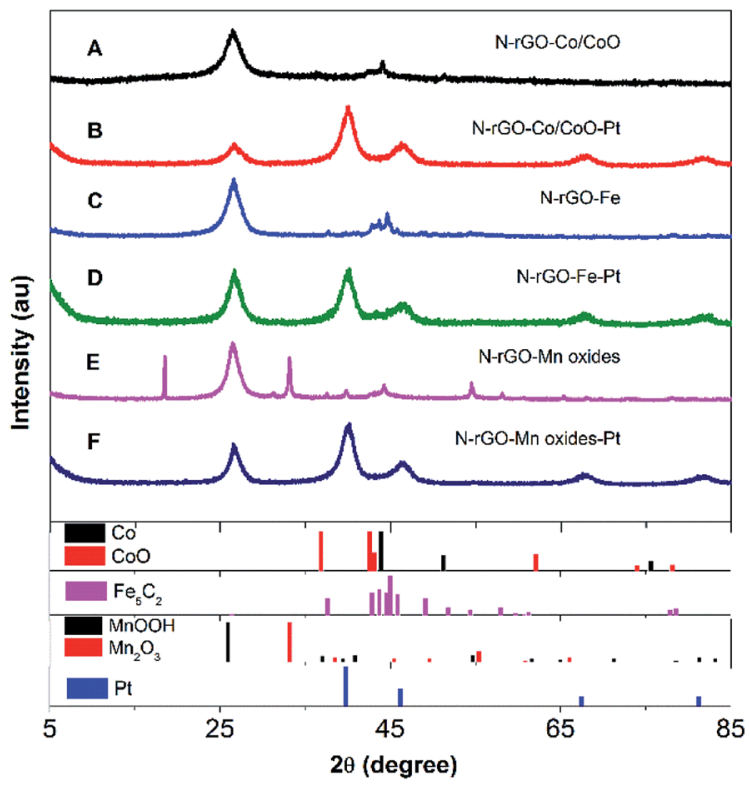

Fig. 1 XRD patterns of (A) N-rGO-Co/CoO, (B) N-rGO-Co/CoO/Pt, (C) N-rGO-Fe, (D) N-rGO-Fe-Pt, (E) N-rGO-Mn oxides and (F) N$\mathrm{rGO}-\mathrm{Mn}$ oxides $-\mathrm{Pt}$.
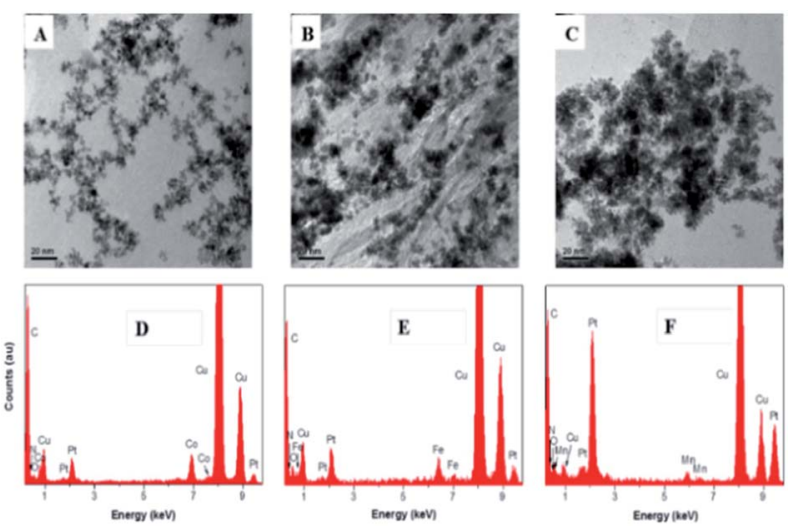

Fig. 2 TEM images of (A) N-rGO-Co/CoO-Pt, (B) N-rGO-Fe-Pt and (C) N-rGO-Mn oxides-Pt and EDS spectra of (D) N-rGO-Co/CoO-Pt, (E) N-rGO-Fe-Pt and (F) N-rGO-Mn oxides-Pt.

and $\mathrm{Mn}$ oxides were efficiently deposited on the graphene surface.

Furthermore, after Pt deposition on the three composites, XRD patterns of the composites showed strong diffraction peaks at $2 \theta=40.2^{\circ}, 46.3^{\circ}, 67.8^{\circ}$ and $81.2^{\circ}$, which are assignable to typical face-centered-cubic (111), (200), (220) and (311) crystalline planes of Pt, respectively (Fig. 1B, D and E). This fact indicates the absence of cobalt ions, iron oxide and manganese oxides diffractions was most likely due to the low crystallinity of the transition metal oxides and the heavy atom effect from platinum. Good dispersion, few aggregates and proper size of $\mathrm{Pt}$ NPs on $\mathrm{N}-\mathrm{rGO}-\mathrm{Co} / \mathrm{CoO}$, N-rGO-Fe and N-rGO-Mn oxides would bestow them with outstanding catalytic performance.

To gain more morphology information about N-rGO-Co/ CoO-Pt, N-rGO-Fe-Pt and N-rGO-Mn oxides-Pt, the TEM images and EDS spectra were characterized (Fig. 2). Fig. 2A shows spherical $\mathrm{Co} / \mathrm{CoO}-\mathrm{Pt}$ NPs are uniformly decorated on $\mathrm{N}$ rGO surfaces, and they are fairly monodispersed, with an average diameter of $4-6 \mathrm{~nm}$. The relatively even distribution and size of Pt nanoparticles may be ascribed to a large number of $\mathrm{Co} / \mathrm{CoO}$ NPs and defects of N-rGO, which not only provide many anchor sites for the uniform deposition of Pt nanoparticles but also enhance the interaction among Pt nanoparticles, $\mathrm{Co} / \mathrm{CoO}$ NPs and N-rGO by coordination interaction as discussed above, ${ }^{25}$ indicating $\mathrm{N}, \mathrm{Co}, \mathrm{O}$ and $\mathrm{Pt}$ components, which is further confirmed by EDS and HRTEM measurements (Fig. 2D and S1, ESI $\dagger$ ). Besides, the phenomena of N-rGO-Fe-Pt and N-rGO-Mn oxides-Pt are the same as that of N-rGO-Co/ CoO-Pt. The structure has shown good electrical conductivities due to the electron hopping between different valence states of metals in O-sites and should also provide necessary surface redox active metal centers for $\mathrm{O}_{2}$ adsorption and activation.

Raman spectroscopy is a useful tool for the characterization of carbon structure of N-rGO-Co/CoO, N-rGO-Fe and N-rGOMn oxides materials. As depicted in Fig. 3, the two prominent peaks at 1355 and $1585 \mathrm{~cm}^{-1}$ are the characteristic peaks of D and $\mathrm{G}$ bands from N-rGO. The intensity ratio $\left(r=I_{\mathrm{D}} / I_{\mathrm{G}}\right)$ of the well-documented $\mathrm{D}$ band and $\mathrm{G}$ band of $\mathrm{N}-\mathrm{rGO}-\mathrm{Co} / \mathrm{CoO}(2.98)$, $\mathrm{N}-\mathrm{rGO}-\mathrm{Fe}$ (3.66) and N-rGO-Mn oxides (4.40) was enhanced, 


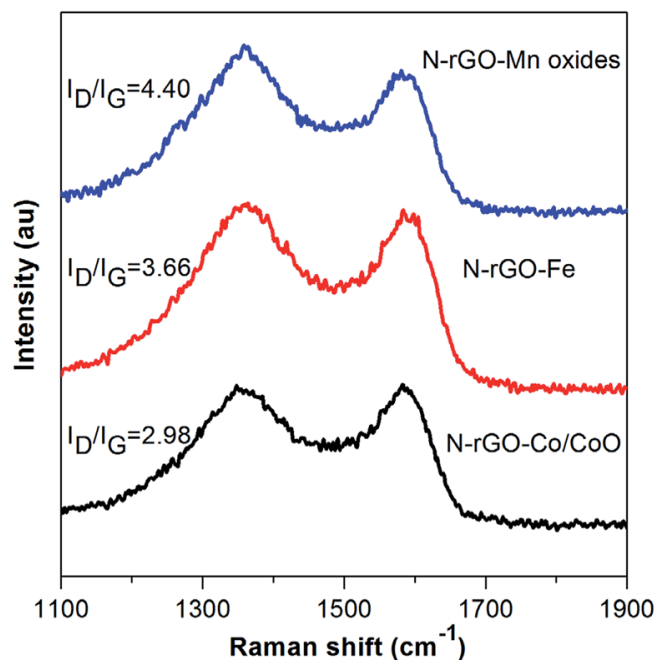

Fig. 3 Raman spectra of N-rGO-Co/CoO, N-rGO-Fe and N-rGOMn oxides.

compared with that of $\mathrm{N}-\mathrm{rGO}(r=1.27)$, (i) defects due to nitrogen doping onto the conjugated carbon framework and (ii) disorder of rGO layers. The $\mathrm{G}$ band of $\mathrm{N}-\mathrm{rGO}-\mathrm{Co} / \mathrm{CoO}, \mathrm{N}-\mathrm{rGO}-\mathrm{Fe}$ and $\mathrm{N}-\mathrm{rGO}-\mathrm{Mn}$ oxides shifted to $1590 \mathrm{~cm}^{-1}$, due to nitrogen doped or strain effect. Furthermore, compared to the N-rGO, the peaks can be found for both $\mathrm{D}$ and $\mathrm{G}$ bands, hinting a significant charge transfer between N-rGO sheets and $\mathrm{M}(\mathrm{MO})$ nanoparticles, which is helpful for improving their electrochemical performance. In the case of $\mathrm{N}-\mathrm{rGO}-\mathrm{Co} / \mathrm{CoO}, \mathrm{N}-\mathrm{rGO}-\mathrm{Fe}$ and $\mathrm{N}$ rGO-Mn oxides, a $4-6 \mathrm{~cm}^{-1}$ red shift of both $\mathrm{D}$ and $\mathrm{G}$ bands was observed. It is generally accepted that the relative intensity ratio of these bands is a measure of the disorder and extent of defects in the graphitic materials, which is favorable for the further electrochemical processes.

In order to obtain more information of the nitrogen, cobalt and oxygen functionalities after codoping processes, $\mathrm{N}-\mathrm{rGO}-\mathrm{Co} /$ CoO-Pt composites were analyzed by XPS in the region of $0-$ $1300 \mathrm{eV}$ (Fig. 4A). The $\mathrm{N}$ 1s spectra of N-rGO-Co/CoO-Pt can be fitted into three peaks at 398.9, 399.9, and $401.4 \mathrm{eV}$ (Fig. 4B, Table 1). The peaks with lower binding energy located at about 398.9 and $399.9 \mathrm{eV}$, respectively, correspond to pyridine-like and pyrrole-like nitrogen, which can contribute to the $\pi$ conjugated system with a pair of p-electrons in the graphene layers. When carbon atoms within the graphene layers are substituted by nitrogen atoms in the form of "graphitic" nitrogen, the corresponding peak in the high-resolution $\mathrm{N} 1 \mathrm{~s}$ spectra is located at $401.4 \mathrm{eV}$. The results demonstrate the quantity of nitrogen precursor melamine and high temperature were the key factor to modulate the chemical state of nitrogen in doped graphene. The Co $2 \mathrm{p}$ XPS spectra of the composite exhibit two peaks at 779.8 and $795.4 \mathrm{eV}$, corresponding to the Co $2 \mathrm{p}_{1 / 2}$ and Co $2 \mathrm{p}_{3 / 2}$ spin orbit peaks of $\mathrm{Co} / \mathrm{CoO}$ (Fig. $4 \mathrm{C}$ ). Co and $\mathrm{CoO}$ spectra are presented in Fig. 4 with spectral fitting parameters given in Table 1, which suggests the fractions of $38.6 \%$ and $61.4 \%$ for Co and CoO, respectively. Additionally, the higher binding energy $2 \mathrm{p}_{3 / 2}$ multiplet or satellite structures of the $\mathrm{CoO}$ with the Co $2 \mathrm{p}_{1 / 2}$ peak at $795.4 \mathrm{eV}$ maybe effectively cause a slight overestimation of the metal, compared to the oxide component. While the peak at $531.7 \mathrm{eV}$ is corresponded to the oxygen species in the $\mathrm{CoO}$ phase, that at $533.0 \mathrm{eV}$ are assignable to the residual oxygen containing groups (such as $\mathrm{OH}$ and $\mathrm{COOH}$ ) on the surface of graphene (Fig. 4D). The XPS spectra of N-rGO-Mn oxides-Pt also confirmed the nitrogen, manganese and oxygen functionalities (Fig. S2, ESI $\dagger$ ). The XPS
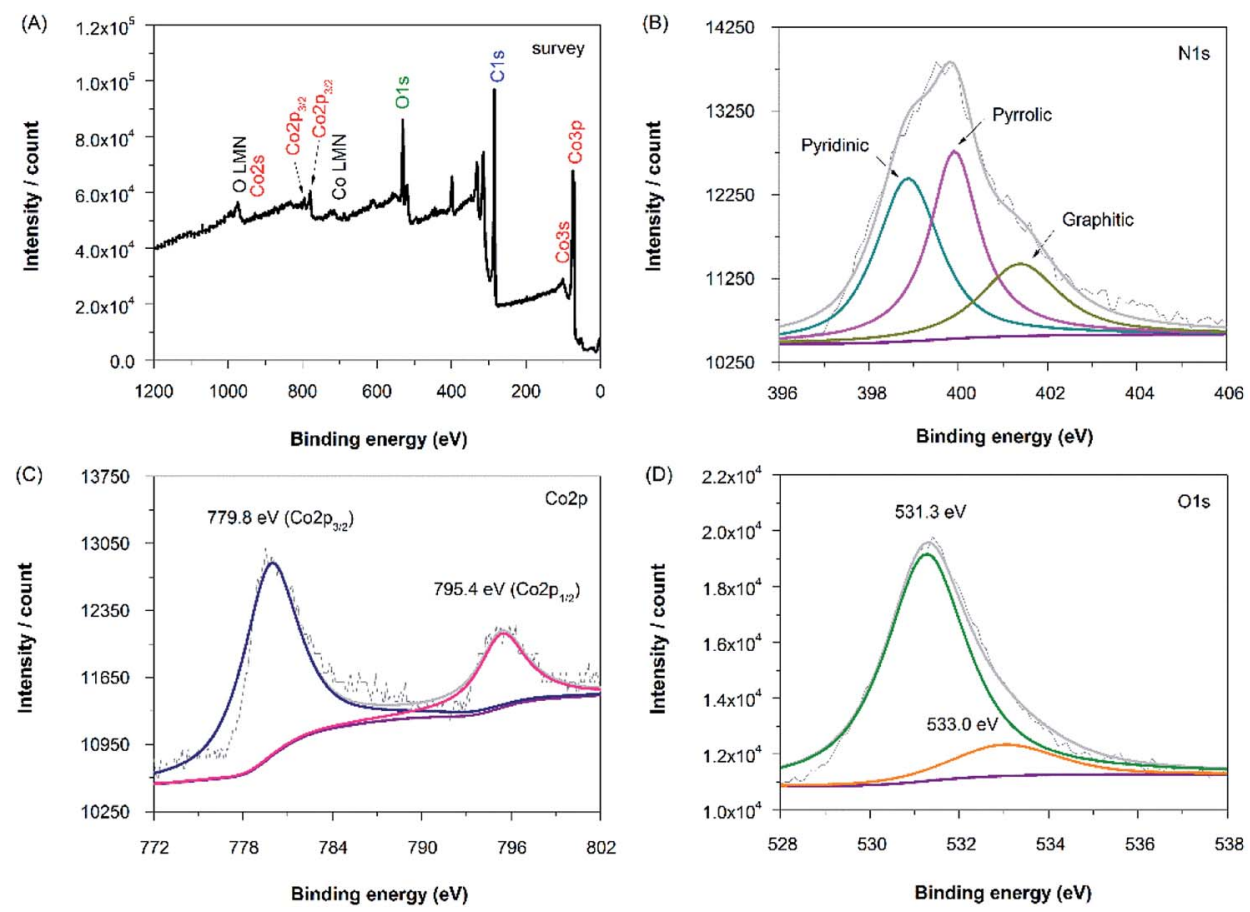

Fig. 4 XPS spectra of the (A) survey scan, (B) N 1s region, (C) Co $2 p$ region and (D) O 1s region of the $\mathrm{N}-\mathrm{rGO}-\mathrm{Co} / \mathrm{CoO}-\mathrm{Pt}$. 
Table 1 Co $2 p_{3 / 2}$ spectral fitting parameters: binding energy (eV) and percentage of total area

\begin{tabular}{|c|c|c|c|c|c|c|c|c|}
\hline Compound & $\begin{array}{l}\text { Peak } 1 \\
(\mathrm{eV})\end{array}$ & $\%$ & $\begin{array}{l}\text { Peak } 2 \\
(\mathrm{eV})\end{array}$ & $\%$ & $\begin{array}{l}\text { Peak } 3 \\
(\mathrm{eV})\end{array}$ & $\%$ & $\begin{array}{l}\text { Peak } 4 \\
(\mathrm{eV})\end{array}$ & $\%$ \\
\hline Co & 778.2 & 38.5 & 781.1 & 38.5 & 783.1 & 23.0 & - & - \\
\hline $\mathrm{CoO}$ & 779.8 & 70.0 & 782.1 & 11.3 & 785.5 & 9.0 & 786.5 & 9.6 \\
\hline
\end{tabular}

spectra of N-rGO-Fe-Pt were not characterized, due to the magnetic properties of Fe.

To assess the catalytic activity of N-rGO-Co/CoO-Pt, N-rGOFe-Pt and N-rGO-Mn oxides-Pt catalysts for ORR, CV and measurements on a RDE were conducted. Apparently, N-rGOCo/CoO-Pt, N-rGO-Fe-Pt and N-rGO-Mn oxides-Pt exhibited a pronounced electrocatalytic ORR activity associated with a more positive ORR onset potential $(-0.15 \mathrm{~V},-0.17 \mathrm{~V}$ and $-0.13 \mathrm{~V}$ relative to $\mathrm{Ag} / \mathrm{AgCl})$, similar to the $20 \% \mathrm{Pt} / \mathrm{C}(-0.12 \mathrm{~V})$ (Fig. 5A), suggesting synergistic ORR catalytic activity of N-rGO, $\mathrm{M}(\mathrm{MO})$ and Pt nanoparticles in the composites. ${ }^{31,32}$

To examine the reaction kinetics for $\mathrm{N}-\mathrm{rGO}-\mathrm{Co} / \mathrm{CoO}-\mathrm{Pt}, \mathrm{N}-$ rGO-Fe-Pt and N-rGO-Mn oxides-Pt, linear sweep voltammograms (LSVs) were further recorded in an $\mathrm{O}_{2}$-saturated $0.1 \mathrm{M}$ $\mathrm{KOH}$ electrolyte at a scan rate of $10 \mathrm{mV} \mathrm{s}^{-1}$ using a rotating disk electrode (RDE) (Fig. 5B). Among these prepared freestanding catalysts, N-rGO-Co/CoO-Pt, N-rGO-Fe-Pt and N-rGO-Mn oxides-Pt showed an enhanced ORR performance (Table 2) with more positive onset potential $(-0.014,-0.040$ and $0.073 \mathrm{~V})$ and large current density $\left(J_{\mathrm{k}}=8.00,1.49\right.$ and $9.11 \mathrm{~mA} \mathrm{~cm}^{-2}$, respectively). The half-wave potential at $1600 \mathrm{rpm}$ was -0.17 , -0.39 and $-0.22 \mathrm{~V}$, respectively, almost more positive than of $\mathrm{Pt} / \mathrm{C}(-0.27 \mathrm{~V})$. This is contributed to the synergistic effects of three components and the interpenetrated network structure in combination Pt, $\mathrm{M}(\mathrm{MO})$ nanoparticles with N-rGO, effectively increasing basal spacing and electrical conductivity. Furthermore, RDE curves of N-rGO-Co/CoO-Pt, N-rGO-Fe-Pt and NrGO-Mn oxides-Pt at various rotation speeds were measured to determine its ORR kinetic performance in $0.1 \mathrm{M} \mathrm{KOH}$ (Fig. 5CE). ${ }^{33}$ The linearity and parallelism of the $\mathrm{K}-\mathrm{L}$ plots suggested reaction kinetics toward the concentration of dissolved oxygen and similar electron transfer numbers for ORR at different potentials (Fig. 5C-E inset). The electron transfer numbers $(n)$ of N-rGO-Co/CoO-Pt, N-rGO-Fe-Pt and N-rGO-Mn oxides-Pt were calculated to be $3.83,3.98$ and 4 , respectively, at $-0.4 \mathrm{~V}$ from the slopes of Koutecky-Levich plots, indicated those materials can favour $\sim 4 \mathrm{e}$ oxygen reduction process, similar to that of $20 \% \mathrm{Pt} / \mathrm{C}$ catalyst $\left(n \sim 4\right.$ for Pt/C). ${ }^{34}$ The electrochemical impedance spectra of the electrode prepared with $\mathrm{N}-\mathrm{rGO}-\mathrm{Co} /$ CoO-Pt, N-rGO-Fe-Pt, N-rGO-Mn oxides-Pt and 20\% Pt/C was shown in Fig. S3. $\dagger$ The low resistance of N-rGO-Co/CoO-Pt, NrGO-Fe-Pt, N-rGO-Mn oxides-Pt and 20\% Pt/C (Table S1, ESI $\dagger$ ) indicates its better charge conductivity, which may arise from heteroatom-doping induced charge transfer in carbon and the high electrical conductivity of those metal materials.

In addition to, the long-term stability of catalysts is one of the major concerns in microbial fuel cell technology. The

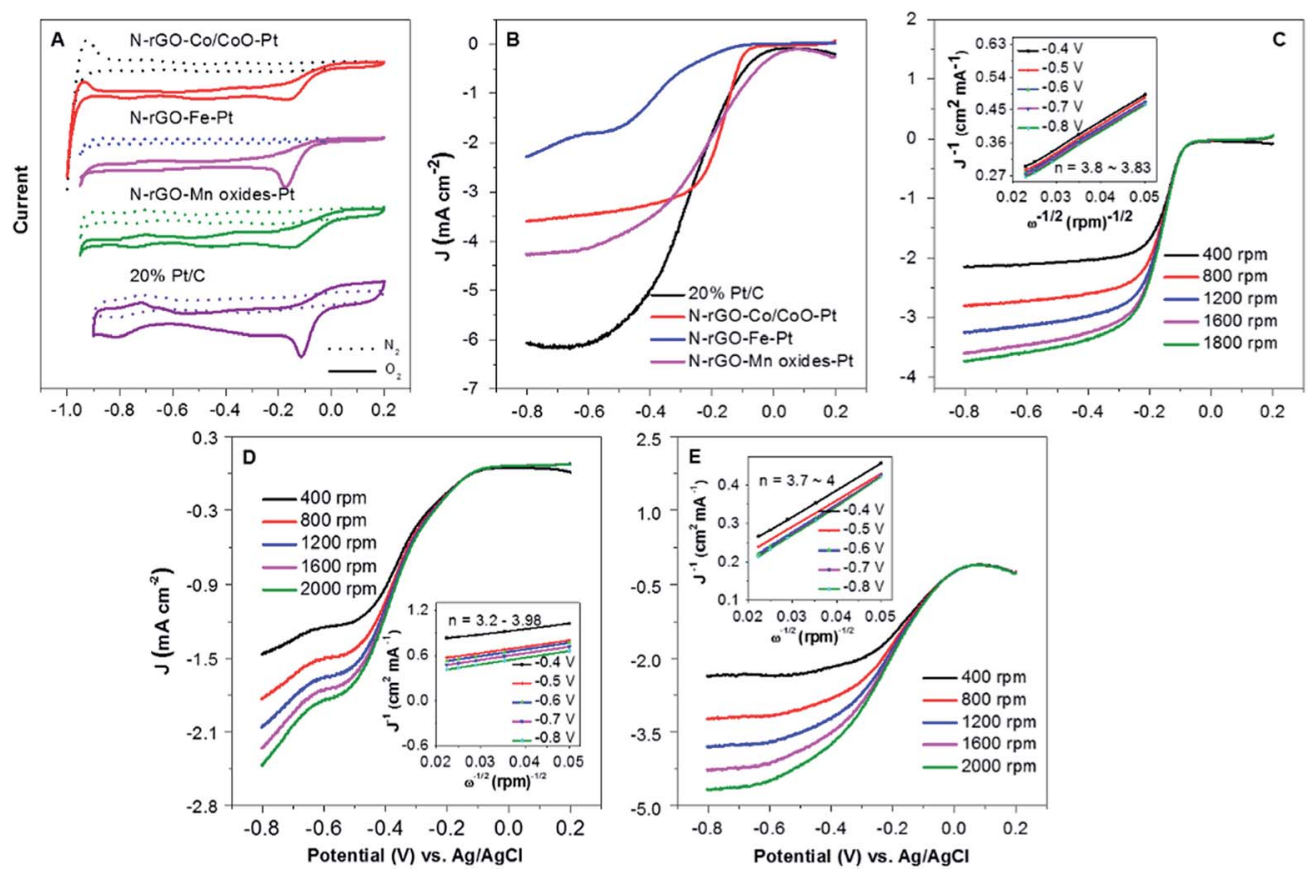

Fig. 5 (A) $\mathrm{CV}$ curves of the $\mathrm{N}-\mathrm{rGO}-\mathrm{Co} / \mathrm{CoO}-\mathrm{Pt}, \mathrm{N}-\mathrm{rGO}-\mathrm{Fe}-\mathrm{Pt}$ and $\mathrm{N}-\mathrm{rGO}-\mathrm{Mn}$ oxides-Pt on glassy carbon electrodes in $\mathrm{O}_{2}$-saturated (solid line) and $\mathrm{N}_{2}$-saturated (dot line) $0.1 \mathrm{M} \mathrm{KOH}$ with scan rate of $10 \mathrm{mV} \mathrm{s}^{-1}$. (B) Linear sweep voltammetry (LSV) for the N-rGO-Co/CoO-Pt, N-rGO$\mathrm{Fe}-\mathrm{Pt}, \mathrm{N}-\mathrm{rGO}-\mathrm{Mn}$ oxides-Pt and $20 \% \mathrm{Pt} / \mathrm{C}$ in $\mathrm{O}_{2}$-saturated $0.1 \mathrm{M} \mathrm{KOH}$ with a sweep rate of $10 \mathrm{mV} \mathrm{s}^{-1}$ at $1600 \mathrm{rpm}$. (C-E) LSV curves of N-rGO$\mathrm{Co} / \mathrm{CoO}-\mathrm{Pt}, \mathrm{N}-\mathrm{rGO}-\mathrm{Fe}-\mathrm{Pt}$ and $\mathrm{N}-\mathrm{rGO}-\mathrm{Mn}$ oxides-Pt in $\mathrm{O}_{2}$-saturated electrolytes at different rotation rates (in rpm), the inset shows the corresponding Koutecky-Levich plot of N-rGO-Co/CoO-Pt, N-rGO-Fe-Pt and N-rGO-Mn oxides-Pt derived from the RDE curves at -0.4$0.8 \mathrm{~V}$ with the electron transport number $(n)$. 
Table 2 Summary of ORR performance of different samples

\begin{tabular}{|c|c|c|c|c|}
\hline & On set potential (V) & Half-wave potential (V) & $J_{\mathrm{k}}\left(\mathrm{mA} \mathrm{cm} \mathrm{cm}^{-2}\right)$ & $n$ \\
\hline $\mathrm{N}-\mathrm{rGo}-\mathrm{Co} / \mathrm{CoO}-\mathrm{Pt}$ & -0.014 & -0.17 & 8.00 & 3.83 \\
\hline N-rGo-Fe-Pt & -0.040 & -0.39 & 1.49 & 3.98 \\
\hline $20 \% \mathrm{Pt} / \mathrm{C}$ & 0.030 & -0.27 & 6.4 & 4 \\
\hline
\end{tabular}

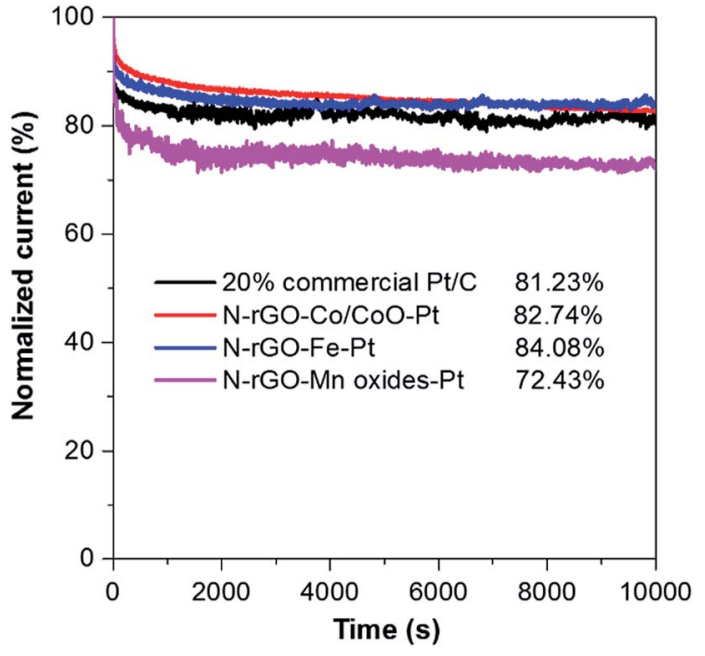

Fig. 6 Chronoamperometric responses of $\mathrm{N}-\mathrm{rGO}-\mathrm{Co} / \mathrm{CoO}-\mathrm{Pt}, \mathrm{N}$ rGO-Fe-Pt, N-rGO-Mn oxides-Pt and $20 \%$ commercial Pt/C modified $\mathrm{GC}$ electrodes at $-0.35 \mathrm{~V}$ with the speed at $1600 \mathrm{rpm}$ in $\mathrm{O}_{2}-$ saturated $0.1 \mathrm{M} \mathrm{KOH}$.

stability of N-rGO-Co/CoO-Pt, N-rGO-Fe-Pt, N-rGO-Mn oxides$\mathrm{Pt}$ and $\mathrm{Pt} / \mathrm{C}$ catalysts was tested at a constant voltage of $-0.35 \mathrm{~V}$ (vs. $\mathrm{Ag} / \mathrm{AgCl}$ ) for $10000 \mathrm{~s}$ in $\mathrm{O}_{2}$-saturated $0.1 \mathrm{M} \mathrm{KOH}$ (Fig. 6). The chronoamperometric response of $\mathrm{N}-\mathrm{rGO}-\mathrm{Co} / \mathrm{CoO}-\mathrm{Pt}$ and $\mathrm{N}$ rGO-Fe-Pt under alkaline conditions, retained a higher relative current of $\sim 82.74 \%$ and $84.08 \%$, respectively, compared to $20 \%$ $\mathrm{Pt} / \mathrm{C}(81.23 \%)$. The better durability of N-rGO-Co/CoO-Pt and NrGO-Fe-Pt can be ascribed to the unique confined structure of $\mathrm{Co} / \mathrm{CoO}$ and Fe NPs within the graphene layers, which can enhance their interfacial contact, suppress the dissolution/ agglomeration of $\mathrm{Co} / \mathrm{CoO}$ and FeNPs, and facilitate the transport of electrolyte ions. However, the chronoamperometric response of $\mathrm{N}-\mathrm{rGO}-\mathrm{Mn}$ oxides-Pt retained a lower relative current of $\sim 72.43 \%$ which could be attributed to the instability of the Mn oxides in alkaline solution.

\section{Conclusions}

We developed a simple high-temperature method to synthesize a N-rGO-M(MO) composite on $\mathrm{N}$-doped reduced graphene oxide, which was further loaded with Pt nanoparticles. Those results indicated that $\mathrm{N}-\mathrm{rGO}-\mathrm{Co} / \mathrm{CoO}-\mathrm{Pt}, \mathrm{N}-\mathrm{rGO}-\mathrm{Fe}-\mathrm{Pt}$ and $\mathrm{N}-$ rGO-Mn oxides-Pt showed excellent electrocatalytic activity for the ORR in alkaline electrolytes, such as a higher current density, higher electron transfer number $(\sim 4)$, and better durability. This resulted from synergistic effects of three components and the interpenetrated network structure in combinations of Pt, M(MO) nanoparticles with N-rGO. Therefore, the synthetic strategy of composites may be further extended to develop other metal or metal oxides/graphenebased monolithic materials for various applications, such as sensors, batteries, and supercapacitors.

\section{Conflicts of interest}

There are no conflicts to declare.

\section{Acknowledgements}

We acknowledge National Natural Science Foundation of China (Project No. 21203118).

\section{Notes and references}

1 F. Cheng and J. Chen, Chem. Soc. Rev., 2012, 41, 2172-2192.

2 K. Kamiya, R. Kamai, K. Hashimoto and S. Nakanishi, Nat. Commun., 2014, 5, 5040.

3 H. Zeng, W. Wang, J. Li, J. Luo and S. Chen, ACS Appl. Mater. Interfaces, 2018, 10, 8721-8729.

4 T. Li, Y. Lu, S. Zhao, Z.-D. Gao and Y.-Y. Song, J. Mater. Chem. A, 2018, 6, 3730-3737.

5 W. Wang, L. Kuai, W. Cao, M. Huttula, S. Ollikkala, T. Ahopelto, A.-P. Honkanen, S. Huotari, M. Yu and B. Geng, Angew. Chem., Int. Ed., 2017, 56, 14977-14981.

6 S. Zhang, Y. Zhang, W. Jiang, X. Liu, S. Xu, R. Huo, F. Zhang and J.-S. Hu, Carbon, 2016, 107, 162-170.

7 R. Huo, W.-J. Jiang, S. Xu, F. Zhang and J.-S. Hu, Nanoscale, 2014, 6, 203-206.

8 M. Luo, Y. Sun, X. Zhang, Y. Qin, M. Li, Y. Li, C. Li, Y. Yang, L. Wang, P. Gao, G. Lu and S. Guo, Adv. Mater., 2018, 30, 1705515.

9 J. Greeley, I. E. L. Stephens, A. S. Bondarenko, T. P. Johansson, H. A. Hansen, T. F. Jaramillo, J. Rossmeisl, I. Chorkendorff and J. K. Nørskov, Nat. Chem., 2009, 1, 552-556.

10 P. Hernandez-Fernandez, F. Masini, D. N. McCarthy, C. E. Strebel, D. Friebel, D. Deiana, P. Malacrida, A. Nierhoff, A. Bodin, A. M. Wise, J. H. Nielsen, T. W. Hansen, A. Nilsson, I. E. Stephens and I. Chorkendorff, Nat. Chem., 2014, 6, 732-738.

11 C.-T. Hsieh, Y.-Y. Liu, D.-Y. Tzou and W.-Y. Chen, J. Phys. Chem. C, 2012, 116, 26735-26743. 
12 S. Pedireddy, H. K. Lee, W. K. Tjiu, I. Y. Phang, R. H. Tan, S. Q. Chua, C. Troadec and X. Y. Ling, Nat. Commun., 2014, 5, 4957.

13 R. Bashyam and P. Zelenay, Nature, 2006, 443, 63-69.

14 J. B. Luo, L. Wang, D. Mott, P. N. Njoki, Y. Lin, T. He, Z. Xu, B. N. Wanjana, I.-I. S. Lim and C.-J. Zhong, Adv. Mater., 2008, 20, 4342-4347.

15 A. Holewinski, J.-C. Idrobo and S. Linic, Nat. Chem., 2014, 6, 828-834.

16 Y. Ito, H. J. Qiu, T. Fujita, Y. Tanabe, K. Tanigaki and M. W. Chen, Adv. Mater., 2014, 26, 4145-4150.

17 J. H. Lee, N. Park, B. G. Kim, D. S. Jung, K. Im, J. Hur and J. W. Choi, ACS Nano, 2013, 7, 9366-9374.

18 H.-W. Liang, X. Zhuang, S. Brüller, X. L. Feng and K. Müllen, Nat. Commun., 2014, 5, 3312.

19 T. Horita, K. Yamaji, M. Ishikawa, N. Sakai, H. Yokokawa, T. Kawada and T. Kato, J. Electrochem. Soc., 1998, 145, 3196-3202.

20 S.-S. Li, H.-P. Cong, P. Wang and S.-H. Yu, Nanoscale, 2014, 6, 7534-7541.

21 J. Liang, R. F. Zhou, X. M. Chen, Y. H. Tang and S. Z. Qiao, Adv. Mater., 2014, 26, 6074-6079.

22 Y. Meng, X. Zou, X. Huang, A. Goswami, Z. Liu and T. Asefa, Adv. Mater., 2014, 26, 6510-6516.

23 X. C. Dong, H. Xu, X. W. Wang, Y. X. Huang, M. B. ChanPark, H. Zhang, L. H. Wang, W. Huang and P. Chen, ACS Nano, 2012, 6, 3206-3213.
24 D. A. C. Brownson, L. C. S. Figueiredo-Filho, X. Ji, M. GómezMingot, J. Iniesta, O. Fatibello-Filho, D. K. Kampouris and C. E. Banks, J. Mater. Chem. A, 2013, 1, 5962-5972.

25 Y. Liang, Y. Li, H. Wang, J. Zhou, J. Wang, T. Regier and H. Dai, J. Am. Chem. Soc., 2012, 134, 3517-3523.

26 Y. Liang, H. Wang, P. Diao, W. Chang, G. Hong, Y. Li, M. Gong, L. Xie, J. Zhou and J. Wang, J. Am. Chem. Soc., 2012, 134, 15849-15857.

27 Z. Yang, X. Zhou, Z. Jin, Z. Liu, H. Nie, X. Chen and S. Huang, Adv. Mater., 2014, 26, 3156-3161.

28 Z. Wang, S. Xiao, Z. Zhu, X. Long, X. Zheng, X. Lu and S. Yang, ACS Appl. Mater. Interfaces, 2015, 7, 4048-4055.

29 Y. Liang, Y. Li, H. Wang, J. Zhou, J. Wang, T. Regier and H. Dai, Nat. Mater., 2011, 10, 780-786.

30 S. Fu, C. Zhu, J. Song, M. Engelhard, H. Xia, D. Du and Y. Lin, ACS Appl. Mater. Interfaces, 2016, 8, 22196-22200.

31 W. Wei, Y. Tao, W. Lv, F. Y. Su, L. Ke, J. Li, D. W. Wang, B. Li, F. Kang and Q. H. Yang, Sci. Rep., 2014, 4, 6289.

32 Z. Wu, K. Parvez, A. Winter, H. Vieker, X. Liu, S. Han, A. Turchanin, X. Feng and K. Müllen, Adv. Mater., 2014, 26, 4552-4558.

33 Z. Wang, R. Jia, J. Zheng, J. Zhao, L. Li, J. Song and Z. Zhu, ACS Nano, 2011, 5, 1677-1684.

34 K. Yamamoto, T. Imaoka, W. J. Chun, O. Enoki, H. Katoh, M. Takenaga and A. Sonoi, Nat. Chem., 2009, 1, 397-402. 